\title{
Identificação e notificação de violência contra crianças e adolescentes: limites e possibilidades de atuação de profissionais de saúde
}

\author{
Milene Maria Xavier Veloso* \\ Celina Maria Colino Magalhães** \\ Isabel Rosa Cabral***
}

\begin{abstract}
Resumo
A violência contra crianças e adolescentes é um desafio para área da saúde e os profissionais tem um papel importante nesse contexto. O presente estudo objetivou caracterizar a percepção de profissionais de saúde sobre violência contra crianças e adolescentes e suas dificuldades para o manejo desse fenômeno. Foi realizado um estudo descritivo com 72 profissionais de saúde da atenção básica do município de Belém-Pará-Brasil. Em relação aos tipos de violência a negligência foi a mais referida pelos profissionais $(60,74 \%)$ seguida da violência sexual $(24,14 \%)$, da física $(39,47 \%)$ e a psicológica (34,88\%), porém a mais notificada foi a sexual $(50 \%)$. Em relação à ficha de notificação $(50,00 \%)$ dos participantes disseram que não a conhece e $86,11 \%$ nunca a utilizaram. Os resultados sugerem que há necessidade de capacitação permanente e de condições instrumentais adequadas para fortalecer a atuação dos profissionais de saúde a superarem os desafios que a intervenção em casos de violência exige.

Palavras-chave: violência, crianças e adolescentes, profissionais de saúde
\end{abstract}

\section{Identification and reporting of violence against children and adolescents: limits and possibilities of action of health professionals}

\begin{abstract}
Violence against children and adolescents is a challenge for health and professionals play an important role in this context. This study aimed to characterize the perception of health professionals about violence against children and adolescents and their difficulties in managing this phenomenon. A descriptive study was carried out with 72 health care professionals from the city of Belém-Pará-Brazil. Regarding the types of violence, negligence was the most reported by professionals $(60,74 \%)$ followed by sexual violence $(24,14 \%)$, physical violence $(39,47 \%)$ and psychological violence $(34,88 \%)$. The most reported was sexual (50,00\%). Regarding the notification form $(50,00 \%)$ of the participants said that they did not know it and $86,11 \%$ never used it. The results suggest that there is a need for permanent training and adequate instrumental conditions to strengthen the performance of health professionals to overcome the challenges that intervention in cases of violence requires.
\end{abstract}

Keywords: violence, children and adolescents, health professionals

* Doutorado em Psicologia pela Universidade Federal do Pará. Professora Adjunto IV da Universidade Federal do Pará.

** Doutora em Psicologia Experimental pela Universidade de São Paulo. Professora Titular da Universidade Federal do Pará, coordenadora do Programa de Pós-Graduação em Teoria e Pesquisa do Comportamento. Bolsista Produtividade em Pesquisa 1D.

***Professora Associada da Universidade Federal do Pará. Doutora em Genética e Biologia Molecular pela Universidade Estadual de Campinas. 


\section{Introdução}

A violência contra crianças e adolescente é uma questão de saúde pública de grande relevância pelo impacto que pode causar aos processos desenvolvimentais das pessoas envolvidas (Borges \& Dell'Aglio, 2012; Pluck, Lee, David, Macleod, Spence \& Randolph, 2011). Além disso, a violência é um processo histórico-social e está diretamente relacionada ao campo da saúde, na medida em que esta área tem a função de elaborar estratégias de prevenção, para promover a saúde da população (Minayo, 2007).

Desde 1990, com a promulgação do Estatuto da Criança e do Adolescente, o governo brasileiro busca estratégias para monitoramento da violência contra crianças e adolescentes, política que culminou com a inclusão da violência interpessoal e autoprovocada na Lista de Notificação Compulsória. Desde 2001, foi regulamentado o instrumento para notificação de casos de suspeita ou de confirmação de maus-tratos contra crianças e adolescentes atendidos no Sistema Único de Saúde (Brasil, 2010). Em função disso, os profissionais de saúde vêm se confrontando com novos e desafiadores problemas em sua prática cotidiana, dentre os quais se destacam a identificação de sinais e sintomas de violência contra crianças e adolescentes e seus desdobramentos.

Alguns estudos têm ressaltado os vários obstáculos que interferem na atuação dos profissionais de saúde e da rede de proteção, frente à questão da violência, tais como a desinformação, a negação, o preconceito e o medo de obrigações legais (Andrade, Nakamura, Paula, Nascimento, Bordin \& Martin, 2011; Day, Telles, Zoratto, Azambuja, Machado \& Silveira, 2003; Giordani, Cezar, Campos, Kretzmann \& Kocourek, 2015; Morais, Sales, Rodrigues \& Oliveira, 2016). Também tem sido destacado que apesar do reconhecimento de suas atribuições, os profissionais demonstram dificuldades em manejar os casos por se tratar de violência intrafamiliar/doméstica (Borowsky \& Ireland, 2002; Garbin, Garbin, Moimaz, Saliba, Costa \& Queiroz, 2011; Rossi \& Silva, 2005; Vieira, Silva, Cavalcante \& Deslandes, 2015). Outros estudos sugerem que o conhecimento teórico, em especial sobre legislação, favorece a identificação e a notificação da violência, e por essa razão, ressaltam a importância da capacitação permanente e de atuação em rede para o atendimento e manejo dos casos identificados em sua rotina de trabalho (Bezerra \& Monteiro, 2012; Gonçalves
\& Ferreira, 2002; Garbin, Rovida, Costa \& Garbin,2016; Silva, Lunardi, Silva \& Filho,2009; Veloso, Magalhães, Dell'Aglio, Cabral \& Gomes, 2013).

No município de Belém-Pará, foram registradas no Sistema de Informação de Agravos de Notificação (SINAN-Net), entre os anos de 2009 a 2011, 3.267 casos de violência interpessoal. Foi possível observar que um único serviço de referência para o atendimento de crianças e adolescentes vítimas de violência sexual foi responsável por $100 \%$ das notificações em 2009, 99\% em 2010 e $72 \%$ em 2011. Ou seja, no período investigado foi mínima a participação da atenção básica na notificação de violência (Veloso, Magalhães, Dell'Aglio, Cabral \& Gomes, 2013).

Esse contexto motivou a realização de um estudo para caracterizar a percepção de profissionais de saúde da atenção básica a respeito da violência contra crianças e adolescentes, os fatores que podem interferir para a identificação e notificação da violência e suas atitudes e dificuldades para o manejo desse fenômeno.

\section{Método}

O estudo foi desenvolvido com 72 profissionais da saúde, que atuavam em unidades de atenção básica à saúde de um distrito administrativo em Belém-PA, no período de junho de 2013 a janeiro de 2014. Trata-se de um estudo descritivo e transversal, onde foram incluídos todos os profissionais interessados em participar da pesquisa em sua unidade de saúde de lotação.

A população de Belém é estimada em cerca de 1,4 milhão de habitantes (IBGE, 2010), distribuídos em 71 bairros e organizados em sete distritos administrativos. $\mathrm{O}$ distrito sanitário do Guamá (DAGUA), onde este estudo foi desenvolvido, inclui seis bairros e é o mais populoso dos distritos administrativos de Belém, abrigando uma população aproximada de 402 mil habitantes, atendida por treze unidades municipais de atenção básica, sendo sete dessas integrantes da Estratégia Saúde da Família. A escolha do Distrito DAGUA deu-se também pelo fato de que o referido distrito abriga áreas de grande índice de violência da capital, recebendo atenção dos governos estaduais e federais para o enfrentamento a essa violência.

O levantamento dos dados foi realizado a partir de questionários elaborados pelos autores e pré-testados com profissionais de unidades que não participariam da pesquisa. Continham perguntas categóricas, de multirresposta e questões abertas relacionadas aos seguintes aspectos: 
dados sociodemográficos, conhecimento sobre sinais e sintomas de violência contra crianças e adolescentes, atitudes diante de casos identificados, o processo de notificação e as dificuldades enfrentadas pelo profissional em relação à temática. As variáveis contínuas ou categóricas foram submetidos à estatística descritiva, sendo também investigada a relação entre variáveis, por meio de testes de comparação de frequências, utilizando-se o Programa BioEstat ${ }^{\circledR} 5.3$ (Ayres, Ayres, Ayres \& Santos, 2005).

Para a sistematização das questões abertas, relativas aos sinais e sintomas de violência, foram criadas categorias a partir da análise de conteúdo temático (Bardin, 1979) e sistematizadas por meio do programa NVivo ${ }^{\circledR}$. As categorias definidas foram: 1) sinais evidentes no corpo da vítima (hematomas, marcas, queimaduras, cortes, feridas); 2) sinais relacionados ao comportamento da vítima (isolamento, agressividade); 3) sinais relacionados a sentimentos da vítima (medo, tristeza); 4) sinais relacionados a problemas no desenvolvimento das vítimas e/ ou problemas no desempenho escolar (atraso escolar, dificuldades cognitivas); 5) sinais relacionados ao comportamento dos pais ou responsáveis (ausência, omissão); 6) sinais relacionados a aspectos gerais (adoecimento, maus-tratos); 7) Não respondeu, informou não saber ou deu respostas incongruentes. Posteriormente tais categorias foram submetidas à análise quantitativa. A pesquisa foi aprovada pelo Comitê de Ética e Pesquisa da Universidade Federal do Pará (CAAE no 0078.0.073.073-11).

\section{Resultados}

Dos 72 profissionais de saúde, participantes do estudo, 59 eram do sexo feminino $(81,94 \%)$, com média de idade de 41,70 anos $( \pm 11,73)$, sem diferença segundo o sexo $(\mathrm{p}=0,135)$. As principais categorias de profissionais entrevistados foram da enfermagem $(34,72 \%)$ e da medicina $(27,88 \%)$, os demais pertenciam à odontologia $(12,5 \%)$, serviço social $(12,5 \%)$, psicologia $(6,94 \%)$, nutrição $(5,56 \%)$ e educação física $(1,39 \%)$. A preponderância de profissionais da enfermagem e medicina deve-se ao fato de que $37,5 \%$ dos profissionais integravam a Estratégia Saúde da Família, tendo sido entrevistados todos os profissionais de nível superior das equipes da área estudada. Na Tabela 1 estão descritas as suas principais características sociodemográficas.

Considerando-se as categorias definidas para análise, em relação à violência física, os sinais de violência mais
Tabela 1 Características sociodemográficas de profissionais de saúde atuantes na atenção básica de um distrito administrativo de Belém- PA.

\begin{tabular}{|c|c|c|}
\hline Variáveis sócio- demográficas & $\mathrm{n}(72)$ & $\%$ \\
\hline \multicolumn{3}{|l|}{ Sexo } \\
\hline Masculino & 13 & $18,06 \%$ \\
\hline Feminino & 59 & $81,94 \%$ \\
\hline \multicolumn{3}{|l|}{ Idade } \\
\hline 20 à 30 anos & 16 & $22,22 \%$ \\
\hline 31 à 40 anos & 27 & $37,50 \%$ \\
\hline 41 à 50 anos & 20 & $27,77 \%$ \\
\hline$>50$ & 9 & $12,49 \%$ \\
\hline \multicolumn{3}{|l|}{ Estado Civil } \\
\hline Casado (a) / em união estável & 33 & $45,83 \%$ \\
\hline Solteiro (a) & 32 & $44,44 \%$ \\
\hline Separado (a) / divorciado (a) & 4 & $5,56 \%$ \\
\hline Viúvo (a) & 2 & $2,78 \%$ \\
\hline \multicolumn{3}{|l|}{$\mathrm{N}^{\circ}$ de filhos } \\
\hline Sem filhos & 30 & $41,7 \%$ \\
\hline De 1 a 2 & 27 & $37,5 \%$ \\
\hline$>2$ & 9 & $12,5 \%$ \\
\hline \multicolumn{3}{|l|}{ Graduação } \\
\hline Enfermagem & 24 & $34,72 \%$ \\
\hline Medicina & 20 & $27,78 \%$ \\
\hline Odontologia & 9 & $12,50 \%$ \\
\hline Serviço Social* & 9 & $12,50 \%$ \\
\hline Psicologia* & 5 & $6,94 \%$ \\
\hline Nutrição* & 4 & $5,56 \%$ \\
\hline Educação Física* & 1 & $1,39 \%$ \\
\hline \multicolumn{3}{|l|}{ Tempo de formado } \\
\hline $0-5$ & 18 & $25,00 \%$ \\
\hline $6-10$ & 9 & $12,50 \%$ \\
\hline $11-15$ & 8 & $11,11 \%$ \\
\hline $16-20$ & 4 & $5,56 \%$ \\
\hline $21-25$ & 10 & $13,89 \%$ \\
\hline$>25$ & 18 & $25,00 \%$ \\
\hline \multicolumn{3}{|l|}{ Critério Brasil } \\
\hline A1 & 2 & $2,77 \%$ \\
\hline A2 & 20 & $27,77 \%$ \\
\hline B1 & 32 & $44,44 \%$ \\
\hline B2 & 10 & $13,88 \%$ \\
\hline $\mathrm{C} 1$ & 2 & $2,77 \%$ \\
\hline $\mathrm{C} 2$ & 5 & $6,94 \%$ \\
\hline $\mathrm{D}$ & 1 & $1,38 \%$ \\
\hline
\end{tabular}

*Apenas se aplica para as UBS, uma vez que por ocasião do presente estudo os profissionais ainda não haviam sido contratados para atuação na Estratégia Saúde da Família da referida área. 
referidos espontaneamente pelos profissionais, foram os relacionados aos sinais físicos no corpo da vítima (46,69\%), com ênfase para os hematomas $(28,30 \%)$, seguido de sinais relativos ao comportamento da vítima, citados por $28,19 \%$ dos entrevistados, destacando-se o comportamento agressivo ou retraído. As alterações de comportamento da vítima foram as mais frequentemente referidas como indicadores de violência sexual $(42,02 \%)$ e da violência psicológica (53,03\%), destacando-se a referência ao isolamento. Os sinais relacionados aos sentimentos da vítima, com especial atenção para o medo, também estiveram associados às violências sexual e psicológica. Para a negligência destacaram-se os sinais físicos no corpo $(57,84 \%)$, seguido dos sinais relacionados ao comportamento dos pais e/ou responsáveis (22,05\%).

Em média 94,06\% dos profissionais identificaram corretamente todas as situações de violência descritas no questionário, com maior índice de acerto para as situações retratando a violência sexual $(96,30 \%)$ e, no outro extremo, aqueles referentes à violência física $(86,95 \%)$. As situações de não monitoramento das atividades escolares, punição por não atingir alto desempenho na execução de tarefas (incluindo escolares) e as agressões físicas por falhas ou para disciplinar, não foram identificadas como formas de violência por 5,58\%. Destaca-se também que $14,08 \%$ da amostra tem dúvida em considerar como violência o ato de punir por não atingir alto desempenho na execução de tarefas, incluindo as escolares, assim como o ato de frequentemente gritar, xingar ou ameaçar de castigo $(9,72 \%)$ ou ainda punir para disciplinar $(6,94 \%)$ e permitir ou incentivar uso de roupas com exposição excessiva do corpo da criança $(6,94 \%)$.

Em relação a proporção de profissionais que, durante a sua rotina de trabalho, identificaram sinais de maus tratos contra crianças e adolescentes e suas atitudes frente à questão, apenas $11,11 \%$ dos profissionais afirmaram nunca ter percebido uma criança e ou adolescente como possível vítima de violência, sendo dois profissionais da medicina, quatro da enfermagem, um da psicologia e um da nutrição.

Dos que relataram ter suspeitado de violência ( $\mathrm{n}=$ 64), $18,75 \%$ citaram os quatro tipos de violência investigados e $21,88 \%$ citaram apenas um tipo de violência, mais frequentemente a negligência. Considerando-se o total da amostra ( $\mathrm{n}=72$ ), a negligência foi o tipo de violência mais relatado pelos profissionais $(n=56 ; 77,78 \%$ ), seguido da violência física ( $\mathrm{n}=47 ; 65,28 \%)$, sexual $(\mathrm{n}=29 ; 40,28 \%)$ e psicológica $(\mathrm{n}=24 ; 33,33 \%)$. Apesar da elevada taxa de identificação de negligência, a maioria dos profissionais afirmou não fazer a sua notificação $(76,78 \%)$. A violência sexual foi a que exibiu maior taxa de relato de notificação (58,62\%), seguida da agressão física, com taxa de 46,81\%. A violência psicológica, além de ser a menos percebida, também é a menos notificada (20,83\%).

A atitude mais frequente dos profissionais, diante das situações de violência, foi conversar com a mãe da suposta vítima $(71,60 \%)$, com maiores proporções para a violência sexual e negligência (cerca de $80 \%$ ). Todavia, também foi de ocorrência significativa (e não excludente) o comportamento de conversar com colegas buscando um encaminhamento $(47,78 \%)$, com maior proporção para a violência física $(55,30 \%)$ e psicológica $(50,00 \%)$. Foi pouco mencionado o medo de represália pelo agressor, pela família da vítima ou mesmo o medo de sofrer consequências no trabalho, variando de zero, para a violência psicológica, a 5,67\%, na violência física.

Um total de 29 profissionais referiu ter notificado ao menos um tipo de violência, correspondendo a 45,31\% dos que a identificaram em sua rotina de trabalho. Apesar dessa informação, observou-se que somente nove desses afirmaram conhecer a ficha de notificação (31,03\%). Em relação ao processo de notificação: 90,28\% dos profissionais entrevistados afirmaram ser esse um dever de todo profissional de saúde, todavia, 50,00\% deles não conhecem a ficha de notificação, $86,11 \%$ nunca a utilizaram e $45,83 \%$ deles não sabem se a ficha está disponível na unidade de saúde onde trabalham.

Dentre as justificativas para não notificação da violência a mais frequente foi a decisão de intervir no processo sem envolver outros atores da rede $(36,46 \%)$, o que foi mais frequente para a violência psicológica $(57,89 \%)$ e negligência $(38,10 \%)$. O desconhecimento técnico do processo de notificação foi o motivo alegado por $28,57 \%$ dos profissionais que não notificaram os casos de negligência, 21,05\% quando a violência é psicológica e $20 \%$ em casos de violência física. Outros motivos, menos frequentes, incluíram o encaminhamento para outro profissional (da psicologia ou serviço social) ou para unidade especializada, medo do suposto agressor, alusão de que a violência é uma questão cultural ou um problema familiar. Merece destaque o fato de que $17,71 \%$, equivocadamente, identificaram a notificação como um ato jurídico para instauração de inquérito policial.

A maioria dos profissionais $(54,17 \%)$ afirmou que a temática da violência contra criança e adolescente não era discutida profissionalmente na unidade. Elevada proporção da amostra $(40,28 \%)$ também afirmou desconhecer a existência de um protocolo a ser seguido na unidade de saúde, diante dos casos de violência. 
Quando foi analisada a identificação de violência segundo a função na unidade, observou-se que o profissional da medicina foi o que mais relatou a identificação da violência física $(80,00 \%)$, seguido do profissional da enfermagem $(70,83 \%)$. Em relação a violência sexual o profissional do serviço social foi o que mais a referiu $(77,78 \%)$ seguido dos médicos $(45,00 \%)$. A violência psicológica foi mais percebida pelos psicólogos (60,00\%), seguida dos profissionais do serviço social e da odontologia $(44,44 \%)$. Nos casos de negligência $100,00 \%$ dos profissionais do serviço social e da odontologia a identificaram, seguida do psicólogo (80,00\%). Cabe ressaltar que a frequência de observação de violência sexual pelo profissional do serviço social foi significativamente maior que para os demais profissionais $(\mathrm{G}=6,02 ; \mathrm{p}=0,04)$.

Em relação aos órgãos integrantes da rede de atenção às vítimas de violência, o Conselho Tutelar foi aquele mais citado, com $31,08 \%$, sendo que $50,00 \%$ desses profissionais disseram confiar em sua atuação. O segundo órgão mais citado foi uma unidade de saúde especializada no atendimento às crianças e adolescentes vítimas de violência sexual, referida por $18,79 \%$ dos entrevistados, com elevado índice de confiabilidade (60,71\%). Em terceiro lugar, as delegacias (incluindo as especializadas) foram citadas por $14,09 \%$ com $42,86 \%$ de confiança. Outros órgãos citados foram os serviços de assistência social $(12,84 \%)$ e do sistema judiciário $(8,78 \%)$.

Dos 72 entrevistados, $58,33 \%$ disseram não ter sofrido violência na infância ou adolescência, $26,4 \%$ responderam afirmativamente, $8,4 \%$ disseram não lembrar e 6,94\% optaram por não responder a essa questão. Dos participantes que afirmaram ter sofrido algum tipo de violência, a psicológica aparece como a mais relatada com $57,1 \%$. Não se observou associação entre essa experiência pessoal e identificação da violência $(\mathrm{p}=0,1723)$, apesar de que todos os profissionais que declararam ter sido vítima de violência $(n=19)$ também identificaram violência na rotina de atendimento, o que foi observado somente para $88,08 \%$ daqueles que não relataram ter sofrido violência $(\mathrm{RR}=1,4 ; \mathrm{p}=0,15)$.

\section{Discussão}

Os profissionais reconhecem sua obrigação quanto à notificação da violência, bem como sabem identificar os sinais de violência e os órgãos da rede de proteção à criança e adolescente vítima de violência. Todavia, a temática violência contra crianças e adolescentes não é profissionalmente discutida nas unidades de saúde, os profissionais desconhecem se a ficha de notificação está disponível ou se existe um protocolo a ser seguido, o que impacta nas atitudes dos profissionais quanto à identificação, notificação e intervenção que deve ser realizada nesses casos. A identificação e notificação dos casos de violência contra crianças e adolescentes, portanto, está presente na rotina de trabalho dos profissionais de saúde, mas não é um procedimento padrão, especialmente a notificação.

Por outro lado, embora não se tenha encontrado associação significativa entre ter tido experiência pessoal com violência e a identificação de casos é comum que esse tema desperte em muitos profissionais sentimentos intensos e de difícil manejo. Em função disso, além do aspecto técnico, os profissionais precisam estar preparados psicologicamente para o contato com vítimas de violência, pois muitos sentimentos como raiva, dor, impotência, medo podem interferir no manejo dos casos, ou seja, os aspectos emocionais podem interferir negativamente no desempenho profissional. No entanto, é necessário que o profissional não perca de vista seu compromisso ético e procure estabelecer uma relação de cooperação no trato com as vítimas e seus familiares (Luna, Ferreira \& Vieira, 2010).

A identificação mais frequente da violência física e negligência, aqui observada, é um fato apontado em outros estudos como sendo os tipos mais comumente identificados, por deixarem vestígios mais evidentes de sua presença (Gomes, Junqueira, Silva \& Junger, 2002; Noguchi, Assis \& Santos, 2004). No caso da violência psicológica, sua invisibilidade é comum, pois não deixa marcas físicas que possam "justificar uma intervenção", além de poder ser percebida pelos profissionais como uma forma aceitável de "disciplinar e educar" as crianças e os adolescentes e, portanto, que eles não devem interferir nesses casos (Bannwart \& Brino, 2004; Rolim, Moreira, Gondim, Paz \& Vieira, 2014).

Diante da suspeição de violência pôde-se perceber que os profissionais referiram atitudes semelhantes, independentemente do tipo de violência, sendo mais frequente conversar com os pais e/ou colegas da unidade, buscando encaminhamentos. Acionar os parentes da criança ou do adolescente pode se ser uma conduta importante, porém pode também dificultar à realização de encaminhamentos que protegeriam a criança e/ou adolescente, se este procedimento for realizado de forma isolada. Conversar com os colegas de trabalho pode ser mais adequado, visto que os desdobramentos seriam realizados em parceria com a equipe e com maior retaguarda da rede de serviços (Bannwart \& Brino, 2004). 
Quanto à notificação dos casos, embora a violência sexual tenha sido a menos identificada, esta aparece como a mais notificada, o que indica que essa forma de violência seja menos tolerada. Pode-se inferir que, para a violência sexual os profissionais reconhecem a importância dos outros atores da rede intersetorial e que o serviço de referência, que atende crianças e adolescentes vítimas de violência sexual no município de Belém-PA, tenha credibilidade por parte dos profissionais.

É importante ressaltar que a palavra notificação talvez esteja sendo utilizada pelos profissionais de saúde como sinônimo de "encaminhamento ou denúncia". Portanto, é fundamental debater a distinção conceitual em relação às palavras notificação, denúncia e encaminhamento, a fim de evitar um comportamento de esquiva ou de insegurança do profissional quanto aos procedimentos mais adequados (Ramos \& Teodoro, 2012; Bannwart \& Brino, 2012).

Em Portugal, por exemplo, em casos envolvendo crianças e adolescentes vítimas de violência, o instrumento utilizado para esse fim é chamado de "Ficha de Sinalização para os Cuidados de Saúde Primários, Serviços de Internamento e Consultas Externas Hospitalares" (Leça, Menezes \& Prazeres, 2011). A palavra "sinalização" pode ser mais indicada nesses casos, visto que a palavra notificação, utilizada no Brasil, parece suscitar casos confirmados de violência, além de ser utilizada para outros tipos de agravos à saúde, o que pode gerar dificuldades adicionais quanto ao procedimento no âmbito da saúde.

Deve-se também considerar a questão da concomitância e complexidade entre os tipos de violência e suas formas de expressão. Embora os profissionais de saúde possam ser os primeiros a detectar sinais de possíveis maus-tratos em crianças, não há uma definição universal e empiricamente determinada. Parece crucial que tal definição seja problematizada no contexto em que a família vive a fim de compreender as necessidades de formação dos profissionais para esse tipo de intervenção (Holge-Hazelton \& Tulinius, 2010). Observou-se nas respostas dos profissionais que o mesmo sinal pode ser atribuído para vários tipos de violência, o que demonstra que diferenciá-los ainda é um processo complexo e que os sinais não devem ser considerados de forma isolada.

Há uma diversidade ampla de sinais e sintomas, permeando os diferentes tipos de violência e foi possível perceber que os profissionais associaram de modo adequado o entrelaçamento deles como reveladores de violência em sua rotina de trabalho. Essa característica do fenômeno também pode explicar as diferenças entre as formas de identificação, os procedimentos a serem adotados, assim como as decisões quanto aos encaminhamentos necessários, embora não deva justificar atitudes de omissão, ou de não notificação dos casos (Ramos \& Silva, 2011; Marcon, Tiradentes \& Kato, 2001).

Além disso, ainda que de forma minoritária, parece existir um conflito pessoal dos profissionais de saúde quanto à intervenção em casos de violência, por se considerar tal intervenção uma "invasão à privacidade" das famílias. Em função disso, é necessário que o debate se faça no campo da ética e da cidadania, considerando-se que a omissão em nome da "privacidade das famílias" não parece razoável, pois onde há vitimização não há cidadania. A alegação de falta de conhecimento, ou mesmo a descrença de resolutividade dos casos, por parte da rede de atendimento, não podem justificar a omissão em casos de maus-tratos contra crianças e adolescentes, mesmo quando suspeitos, até porque uma parcela significativa dentre os profissionais, disse confiar nos órgãos da rede do município de Belém-PA.

Outra questão relevante, diz respeito à atuação do Conselho Tutelar, pois foi a organização mais identificada. Aparentemente, a prática adotada pelos profissionais de saúde é, dependendo do caso, comunicar ao Conselho Tutelar sobre o caso, o que está de acordo com as leis e as normas brasileiras. No entanto, os profissionais dizem confiar na atuação do Conselho Tutelar, o que se difere de outros estudos sobre esta temática, que apontam a falta de clareza quanto aos procedimentos adotados pelos Conselhos Tutelares e a desarticulação da rede de proteção, como entraves para que o profissional realize a notificação (Ramos \& Teodoro, 2012; Marcon, Tiradentes \& Kato, 2001)

Outros estudos sugerem que há uma oscilação por parte dos profissionais entre a crença e a descrença da resolubilidade dos casos, além da presença de medo de represálias e insegurança emocional para intervir em casos de violência (Gonçalves \& Ferreira, 2002; Luna, Ferreira \& Vieira, 2010; Gomes, Junqueira, Silva \& Junger, 2002) o que diferiu em parte dos resultados do presente estudo, uma vez que o medo foi pouco referido nas respostas dos profissionais de saúde.

A criação de instrumentos como a ficha de notificação demostra um avanço no enfrentamento da violência contra crianças e adolescentes. Entretanto, a rede de saúde, não têm equipes capacitadas para prestar atendimento adequado às vítimas de violência o que tem gerado uma dissociação entre os preceitos legais e a atuação dos profissionais de saúde diante do assunto, o que também 
foi observado no presente estudo (Bezerra \& Monteiro, 2012; Rolim, Moreira, Gondim, Paz \& Vieira, 2014).

A efetivação de um atendimento depende da possibilidade do profissional de saúde ser capaz de identificar sinais de violência. No entanto, a visibilidade dos sinais depende, dentre outros aspectos, da escuta e do olhar ampliado que o profissional consegue imprimir em seu atendimento. Para tal, os profissionais de saúde precisam de capacitação e educação permanente, todavia trata-se de um desafio que não diz respeito somente ao repasse de informação e de conhecimento sobre a temática, mas, sobretudo, depende da implicação social dos profissionais de saúde a respeito da questão (Rossoni \& Lampert, 2004).

O trabalho em rede exige, por parte dos atores sociais, o exercício permanente de revisão de valores, atitudes e compromissos, devendo se configurar em um processo institucional, e não em ações individuais. Os profissionais de áreas estratégicas como, a educação, a saúde e a área social, são os que trabalham diretamente com uma população em condições de maior vulnerabilidade no país e por esse motivo, é necessário fomentar entre os jovens estudantes dessas áreas a sensibilidade com o dia-a-dia da miséria, da violência, "buscando e dando soluções para a dor de tanta gente com dores tantas". Para isto, "precisamos de perfis profissionais humanistas e críticos" e que isso seja fomentado também na graduação (Ceccim, 2005; Feuerwerker \& Sousa, 2000).

Além disso, é válido perguntar: a atuação frente a violência vai eliminando a condição humana da prática profissional? Nesse sentido, como tornar seres humanos mais humanos, perguntava-se Bronfenbrenner? E ao tentar responder a essa questão ele sugeriu o que chamou de "propostas absurdas". Uma delas seria criar para as futuras gerações o "currículo do cuidar". Segundo ele, "nenhuma sociedade pode se sustentar a não ser que seus membros aprendam as motivações, sensibilidade e habilidades que as demandas de cuidado exigem" (p.272). A finalidade desse currículo não seria aprender a cuidar, mas empenhar-se em fazê-lo (Bronfenbrenner, 2011), o que parece muito pertinente para ser desenvolvido desde muito cedo com todos os profissionais de saúde, seja na graduação ou nas capacitações continuadas.

A atuação dos profissionais de saúde, no enfrentamento da violência, é uma realidade possível, desde que sejam fornecidas as condições instrumentais aos profissionais e esclarecidas suas responsabilidades. Deve-se, portanto, promover uma atuação intersetorial dos profissionais da saúde com a rede de atenção e o Sistema de Garantia de Direitos. Adicionado a isso, é fundamental a inserção da temática nos currículos de graduação, em especial para todos os profissionais da área da saúde, com ênfase para uma atuação interdisciplinar nas equipes de saúde para o enfrentamento de problemas complexos como a violência.

É essencial também que os profissionais desenvolvam métodos para identificar, gerenciar riscos e monitorar os grupos vulneráveis bem como articular ações direcionadas às famílias, aos serviços públicos e organizações em geral, atuando de forma inclusiva e protetora. A intersetorialidade nos serviços de saúde precisa ser efetivada de modo a garantir a qualidade e a continuidade das ações e este é um dos maiores desafios a ser enfrentado.

\section{Referências}

Andrade, E.M., Nakamura, E., Paula, C.S., Nascimento, R., Bordin, I.A. \& Martin, D. (2011). A visão dos profissionais de saúde em relação à violência doméstica contra crianças e adolescentes: um estudo qualitativo. Saúde e Sociedade. São Paulo, 20(1):147-55. doi.org/10.1590/S0104-12902011000100017

Ayres, M., Ayres, J.M., Ayres, D.L. \& Santos, A.S.(2005). BioEstat 5.3: Aplicações estatísticas nas áreas das ciências biológicas e médicas. 5th ed. Belém (PA): Sociedade Civil Mamirauá. CNPq

Bannwart, T.H., Brino, R.F.(2004). Dificuldades enfrentadas para identificar e notificar casos de maus-tratos contra crianças e/ou adolescentes sob a óptica de médicos pediatras. Revista Paulista de Pediatria; 29(2),138-145. doi. org/10.1590/S0103-5822011000200002

Bardin, L.(1979). Análise de conteúdo. Edições 70, Lisboa, 229p.

Bannwart, T. H., \& Brino, R. F.(2012). Maus-tratos contra crianças e adolescentes e o papel dos profissionais de saúde: estratégias de enfrentamento e prevenção, in Habigzang LF, Koller S H. Violência contra crianças e adolescentes Teoria, Pesquisa e Prática. Porto Alegre: Artmed, 280p.

Bezerra, K.P. \& Monteiro, A.I.(2012). Violência intrafamiliar contra a criança: intervenção de Enfermeiros da Estratégia Saúde da Família. Revista da Rede de Enfermagem do Nordeste; 13(2): 354-64. Recuperado: http://www.revistarene.ufc.br/revista/index.php/revista/article/view/219/pdf>

Brasil (2010). Ministério da Saúde. Linha de cuidado para atenção integral à saúde de crianças, adolescentes e suas famílias em situação de violência: orientações para gestores e profissionais de saúde. Secretaria de Atenção à Saúde. Departamento de Ações Programáticas e Estratégicas. Brasília/ DF. 91p.

Borges J.L. \& Dell'Aglio D.D. (2012). Exposição ao abuso sexual infantil e suas repercussões neuropsico-biológicas. In Habigzang L.F., Koller S.H., editores. Violência contra crianças e adolescentes teoria, pesquisa e prática. Porto Alegre: Artmed, 280 p.

Borowsky, I.W., Ireland, M.(2002). Parental screening for intimate partner violence by pediatricians and family physicians. Pediatrics, 110(3), 509-16. Recuperado: https://www.ncbi.nlm.nih.gov/pubmed/12205252.

Bronfenbrenner, U. (2011). Bioecologia do Desenvolvimento Humano: tornando os seres humanos mais humanos. Porto Alegre: Artmed, 310 p.

Ceccim, R.B.(2005). Educação Permanente em Saúde: desafio ambicioso e necessário. Interface Comunicação, Saúde, Educação, 9(6), p.161-77. Recuperado em: http://www.escoladesaude.pr.gov.br/arquivos/File/textos $\% 20$ eps/ educacaopermanente.pdf

Day, V.P.; Telles, L.E.B; Zoratto, P.H.; Azambuja, M.R.F.; Machado, D.A.; Silveira, M.B.; et al.(2003). Violência doméstica e suas diferentes manifestações. Revista Psiquiatria Rio Grande do Sul, 25, Supl 1, 9-21. Recuperado em: http://www.scielo.br/pdf/rprs/v25s1/a03v25s1.pdf

Feuerwerker, L. C. M., \& Sousa, M. F. (2000). Em busca de um novo paradigma: a arte de trabalhar em rede. Divulgação: Em Saúde Para Debate, 21, 49-53.

Garbin, C.A.S., Garbin, A.J.I., Moimaz, S.A.S., Saliba, O., Costa, A.C.O. \& Queiroz, A.P.D.G.(2011). Notificação de violência contra criança: conhe- 
cimento e comportamento dos profissionais de saúde. Revista Brasileira Pesquisa e Saúde, 13(2):17-23. Recuperado em: http://www.periodicos.ufes. br/RBPS/article/viewFile/1597/119

Garbin, C.A.S, Rovida, T.A.S, Costa, A.A. \& Garbin, A.J.I. (2016). Percepção e atitude do cirurgião-dentista servidor público frente à violência intrafamiliar em 24 municípios do interior do estado São Paulo, 2013-2014 Epidemiologia e Serviços de Saúde. Brasília, 25(1):179-186. doi:10.5123/S167949742016000100019

Giordani, J.M.A, Cezar, P.K., Campos, G., Kretzmann, F.G. \& Kocourek, S. (2015). Characteristics of family health professionals in care of violence against children and adolescents. Revista de Enfermagem. UFSM,5(2):316-326. doi.org/10.5902/2179769216375

Gomes R., Junqueira M.F.P., Silva C.O.,\& Junger W.L.(2002). A abordagem dos maus-tratos contra a criança e o adolescente em uma unidade pública de saúde. Ciência \& Saúde Coletiva; 7(2), 275-283. doi.org/10.1590/S141381232002000200008

Gonçalves, H.S. \& Ferreira, A.L.(2002). A notificação da violência intrafamiliar contra crianças e adolescentes por profissionais de saúde. Caderno de Saúde Pública; 8(1): p.315-19. doi.org/10.1590/S0102-311X2002000100032

Holge-Hazelton B., Tulinius C. (2010). Beyond the specific child. What is 'a child's case'in general practice? British Journal of General Practice; 60(570). Recuperado em: https://www.ncbi.nlm.nih.gov/pmc/articles/PMC2801805/

Instituto Brasileiro de Geografia e Estatística. Censo 2010. Rio de janeiro: Acessado em 13 de outubro de 2015 em:// www.ibge.gov.br.

Leça A., Menezes B., Prazeres V.(2011). Maus Tratos em Crianças e Jovens - Guia Prático de Abordagem, Diagnóstico e Intervenção. Direção Geral de Saúde, Portugal.Recuperado:http://www.adcl.org.pt/observatorio/pdf/ Guia_Maus_tratos2011.pdf

Luna G.L.M., Ferreira R.C., Vieira L.J.E.S.(2010). Notificação de maus-tratos em crianças e adolescentes por profissionais da Equipe Saúde da Família. Ciência \& Saúde Coletiva; 15(2): 481-491. doi.org/10.1590/S141381232010000200025

Marcon S.S., Tiradentes L.K., Kato E.S.(2001). Conhecimento, atitudes e crenças de profissionais de saúde de Maringá frente à violência familiar contra a criança e o adolescente. Família Saúde e Desenvolvimento. Curitiba. 3(1):35-47. Recuperado de http://revistas.ufpr.br/refased/article/view/4947/3768

Minayo, M.C.S. (2007). A inclusão da violência na agenda da saúde: trajetória histórica. Ciência Saúde Coletiva, 11supl:1259-67.Recuperado em: http:// www.scielo.br/pdf/csc/v11s0/a15v11s0.pdf

Morais, R.L.G.L., Sales, Z.N., Rodrigues, V.P. Oliveira, J.S.(2016). Ações de proteção a crianças e adolescentes em situação de violência. Revista de Pesquisa Cuidar é Fundamental, Online 8(2):4472-4486. Doi: 10.9789/21755361.2016.v8i2.4472-4486
Noguchi M.S., Assis S.G.\& Santos N.C.(2004). Entre quatro paredes: atendimento fonoaudiológico a crianças e adolescentes vítimas de violência. Ciência \& Saúde Coletiva;9(4):963-973. doi.org/10.1590/S141381232004000400017

Pluck G., Lee K., David R., Macleod D.C., Spence S.A. \& Randolph, W.P.(2011). Neurobehavioural and cognitive function is linked to childhood trauma in homeless adults. British Journal of Clinical Psychology; 50, 33-45. doi: 10.1348/014466510X490253.

Ramos M.L.C.O., Silva A.L.(2011). Estudo sobre a Violência Doméstica Contra a Criança em Unidades Básicas de Saúde do Município de São Paulo - Brasil. Saúde e Sociedade. São Paulo, 20(1), p.136-146. doi.org/10.1590/ S0104-12902011000100016

Ramos M.S., Teodoro M.L.M.(2012). A importância da capacitação dos profissionais da saúde que trabalham com vítimas de violência na infância e adolescência. In Habigzang L.F., Koller S.H. Violência contra crianças e adolescentes teoria, pesquisa e prática. Porto Alegre: Artmed; p. 242-254.

Rolim, A.C.A., Moreira, G.A.R., Gondim S.M.M., Paz, S.S. \& Vieira, L.J.E.S.(2014). Fatores associados à notificação de maus-tratos em crianças e adolescentes realizada por enfermeiros na Atenção Primária à Saúde. Revista Latino Americana de Enfermagem, 22(6):1048. DOI: 10.1590/01041169.0050 .2515

Rossi, D.\& Silva, J.L.P.(2005). Indicadores da violência doméstica praticada contra crianças e adolescentes. Revista Ciência Médica. Campinas, 14(6):495502. Recuperado em http://periodicos.puc-campinas.edu.br/seer/index. $\mathrm{php} /$ cienciasmedicas/article/viewFile/1142/1117

Rossoni E., Lampert L.(2004). Formação de profissionais para o Sistema Único de Saúde e as diretrizes curriculares. Boletim da Saúde, 18 (1), p 87-98. Porto Alegre. Recuperado em file:///D:/Downloads/20140521092044v18n1_ 09formacaoprof.pdf

Silva, P.A., Lunardi, V.L., Silva, M.R.S \& Filho, W.D.L.(2009). A notificação da violência intrafamiliar contra crianças e adolescentes na percepção dos profissionais de saúde. Ciência e Cuidado em Saúde; 8(1), 56-62. doi: 10.4025/ cienccuidsaude.v8i1.7774

Veloso, M.X.V, Magalhães, C.M.C., Dell'Aglio, DD, Cabral, I.R. \& Gomes, M. M.(2013). Notificação da violência como estratégia de vigilância em saúde: perfil de uma metrópole do Brasil. Ciência e Saúde Coletiva. Rio de Janeiro, 18(5), 1263-72. doi.org/10.1590/S1413-81232013000500011.

Vieira, L.J.E.S.; Silva, R.M.S.; Cavalcante, L.F.C., Deslandes, S.F. (2015). Capacitação para o enfrentamento da violência sexual contra crianças e adolescentes em quatro capitais brasileiras. Ciência \& Saúde Coletiva. 20(11):3407-3416. doi.org/10.1590/1413-812320152011.20512014.

Submetido em: 9-11-2016

Aceito em: 16-5-2017 